Vijay Bhatti, Research Associate, Jeremy KenneyHerbert, Consultant Forensic Psychiatrist, Rosemarie Cope, Clinical Director and *Martin Humphreys, Senior Lecturer, Reaside Clinic,
Birmingham Great Park, Bristol Road South, Rubery, Birmingham B45 9BE

*Correspondence

\title{
Using the Health of the Nation Outcome Scales in clinical practice
}

\author{
Michael James and Robert Kehoe
}

\begin{abstract}
Aims and method To describe the implementation of a plan to use a validated outcome measure in the care and treatment of people with severe mental illness within a district general hospltal psychiatric service. Multiple techniques were necessary to promote actual change of practice.

Results A survey of practice found $77 \%$ of full Care Programme Approach patients to have recorded Health of the Nation Outcome Scales (HoNOS) scores in their care plans one year after the beginning of the implementation plan.

Clinical implications it is possible to incorporate the use of HoNOS in to everyday practice but it takes a lot of time, effort and resources. Mental health services may require a clearer indication from the NHS Executtive regarding the use of such outcome scales before committing themselves.
\end{abstract}

The first target of the Health of the Nation mental health strategy was to improve significantly the health and social functioning of mentally ill people (Department of Health, 1992). The Research Unit of the Royal College of Psychiatrists was commissioned to develop an outcome measure to determine such change and the result is a brief assessment tool that assesses physical, personal and social problems associated with mental illness, the Health of the Nation Outcome Scales (HoNOS; Wing et al, 1995). Mental health services have been encouraged by the College to use HoNOS as the standard outcome measure for people with enduring mental illness and HoNOS could be part of a 'minimum data set', if implemented throughout the National Health Service (NHS). HoNOS is already being used in health service research settings (Taylor \& Wilkinson, 1997) and is an integral part of other rating tools being developed such as CORE (Centre for Outcomes Research and Effectiveness; British Psychological Society, 1998).

\section{Using HoNOS in a local district service}

Airedale NHS Trust serves a mixed urban and rural population of 190000 across North and West Yorkshire. Locally, it was recognised by both purchasers and providers that there is a need to measure outcomes in mental health services. To consider the use of HoNOS within the Mental Health Directorate three members of staff attended a training day organised by the Royal College of Psychiatrists: a consultant psychiatrist, a community psychiatric nurse and a community mental health team leader. They would be the key players in planning. organising and delivering the training within the Directorate.

There was an acknowledgement that implementing the use of HoNOS was a long-term project, so a group was established to manage the process, consisting of a consultant psychiatrist, service manager and community mental health team leader.

Three phases were identified for rolling out the programme; training staff who were to carry out ratings, implementing the use of the scales and evaluating its use.

The plan produced by this project group was endorsed by the Directorate Management Group in early 1996 and responsibility for its implementation specified in a manager's objectives. It was recognised that its success required firm commitment from mental health staff, as well as collaborative working with social services. 
There were potential problems in trying to implement the use of the scale. Its use might generate fears or cynicism, especially regarding the use of aggregated data; it may be viewed as yet another new theme, and practitioners may be unwilling or unmotivated to use HoNOS, seeing it as more paperwork.

It was thus felt important to identify the benefits of its introduction such as using a recognised outcome measure which helps in defining needs or deficits in care, targeting services towards the severely mentally ill and providing a more objective outcome measure in individual cases. It was also perceived to be a potentially useful tool in examining case mix. Furthermore, a recent local audit had identified the use of more than fifty different rating scales within the mental health unit and the employment of HoNOS was seen as an opportunity to reduce this number.

\section{Training and implementation}

Local training sessions were organised using the model of the College Research Unit's Trainers course. These covered the background to HoNOS, the structure and scoring of the scales, and the key principles for rating followed by a systematic run through the items and supervised use of the scales using a case study. The sessions were concluded with advice on how to implement their use within service areas. These training sessions lasted 2.5 hours and were facilitated by the clinicians who had attended the oneday College Research Unit course. Within the Airedale mental health unit 130 staff act as keyworkers or primary nurses and following 10 training sessions over eight months, 110 had attended.

Initially, the training sessions were centrally advertised and run, open to mental health staff from the trust and social services. Some clinical areas did implement HoNOS soon after the initial training sessions. However, implementation was patchy across the service and this led to a review.

\section{Trouble-shooting}

It was apparent that a lack of clear guidelines had caused confusion in some areas as to how the use of HoNOS should be implemented. There was also a lack of commitment and ownership from a small number of clinical leaders which needed to be addressed. Implementation was most successful where more than four staff from one clinical area had together attended a training session, and had started using the scales soon after the event. A HoNOS Interest Group was established in early 1997 with a lead individual from each clinical area (e.g. in-patient ward, day hospital, community mental health team); someone who was interested in the use of the outcome scales and in championing their cause. This group acted as a forum to clarify roles and responsibilities. A written protocol was produced, providing guidance on who should carry out the ratings, when the ratings should be performed and which service users/patients should be rated. For example, all adult acute admissions and patients subject to full or complex Care Programme Approach (CPA) have HoNOS scores completed by the primary nurse (for in-patients) or keyworker at specific time intervals such as at each CPA review.

There was a lack of clarity and continuity regarding where HoNOS score results were scored. This was resolved by incorporating a summary of the HoNOS results into the existing CPA documentation.

Training sessions were arranged for specific clinical areas with the intention that the use of the scales would be implemented as soon as possible after the training within those areas. Patients themselves were not always content to have HoNOS scores integrated with their care plans (of which they are given a copy). Several complained about scores on scales of symptoms and aggressive behaviour, either challenging the actual score or complaining about the very presence of such ratings on their care plan. We now keep HoNOS scores separate from the main page of the care plan and avoid sharing its findings with certain patients.

A shorter 'awareness' course has been designed for unqualified staff such as social work aides, nursing assistants and housing support workers. While these care workers will not be directly responsible for performing ratings, it was felt that the provision of information on the scales would assist them in understanding and contributing to the process where ratings are part of the CPA review meeting. They are often the staff members with the highest degree of contact with service users and are thus a valuable source of information.

The HoNOS Interest Group gave added momentum to the implementation process. It provides a forum to discuss and hopefully solve ongoing problems, particularly useful for the lead individuals from the various clinical areas.

\section{Evaluation}

One year after the introduction of the HoNOS protocol to all clinical areas, an audit was performed. In a randomised sample of 73 patients on full CPA from all clinical areas, $77 \%$ of cases had fully accessible recorded scores on 
HoNOS in the care plans, and $54 \%$ had been completed at appropriate time intervals.

Qualitatively, the issue of benefit of using HoNOS has been addressed. Some practitioners find that HoNOS scores are useful in terms of informing individual care plans, and also for the keyworker and patient to review the outcome following a certain intervention. However, other practitioners see little benefit to clinical patient care and complain about 'extra paperwork'.

We have established 3-6 monthly teaching sessions for new staff and HoNOS scores continue to be an integral part of our practice under the CPA.

The costs involved in such a project are high. The main cost is of staff time - both in terms of training and in its everyday use. Initial set-up costs included one day's training for three staff and two went on to be the local trainers. Between five and 10 staff continue to meet on a three monthly basis as the HoNOS Interest Group. If it is assumed that an 'average' mental health professional costs $£ 20 /$ hour, the annual costs of such a project (excluding its everyday usage) would be in the region of £10-15 000/year (for training sessions and HoNOS Interest Group).

\section{Discussion}

To promote change within the health service is never an easy task. Successful change usually requires techniques such as providing information, education, peer review or audit, use of opinion leaders or financial incentives (Stocking, 1992). In the implementation of the use of the HoNOS rating scale we used all of these techniques except financial incentives. Individual professional gain could be added to this list. if we accept that the occasional trip to make a presentation is a pleasant experience. The barriers to change that were met were those typical within a health service setting. Difficulties such as lack of perceived relevance, conflicting priorities, difficulty in measuring success of the project, lack of resources and intensity of contribution have been identified when trying to put evidence into practice (King's Fund, 1998). Other sources have noted that team work is the most effective means of bringing innovation to organisation (Firth-Cozens, 1992) and for the successful use of HoNOS we have found that a team approach is necessary, our training days being reorganised to address this issue.

Although mental health outcome measures are not prominent within a recent NHS Executive publication (1998) Clincial Effectiveness Indicators, there is little doubt that increased emphasis will be put on clinical outcome indicators as well as 'performance' indicators. The introduction of Clinical Governance ensures that all NHS trusts will have to focus on clinical quality issues as well as financial and quantity issues. HoNOS is a practical scale to measure such clinical outcome in mental health.

\section{Conclusions}

It is possible to incorporate the use of a validated rating scale for severe mental illness into everyday practice. However, this seemingly simple change within practice has taken a lot of time. effort and resource. Initial training of a crosssection of staff, clear leadership and endorsement by the management team have assisted in its implementation, but a sizeable proportion of staff (and most likely, users of the service) remain sceptical of its usage because of the lack of direct benefits. We have managed to sustain the regular use of HoNOS for 12-18 months but its longer term usage remains vulnerable. Many mental health services will require a clearer indication from the NHS Executive that the use of HoNOS is 'recommended' or 'required', before investing in the necessary training and implementation process.

\section{References}

BRITISH PSYCHOLOGICAL SOCIETY (1998) A Core Assessment and Outcomes Package for the Care Programme Approach. London: University College London.

DEPARTMENT OF HEALTH (1992) The Health of the Nation London: HMSO.

FIRTH-COzENS, J. (1992) Building teams for effective audit. Quality in Health Care, 1. 252-255.

KING'S FUND (1998) Turning Evidence into Practice Everyday. London: King's Fund Publishing.

NHS EXECUTIVE (1998) Clinical Effectiveness Indicators: A Consultation Document. Leeds: NHS Executive.

STocking, B. (1992) Promoting change in clinical practice. Quality in Health Care, 1, 56-60.

TAYLOR, J. \& WILKINSON, G. (1997) HoNOS $v$. GP opinion in a shifted out-patient clinic. Psychiatric Bulletin, 21. 483-485.

Wing, J., BEAVER, A. S., CuRTIS, R. H., et al (1995) HoNOS Health of the Nation Outcome Scales. London: Royal College of Psychiatrists' Research Unit Report.

Michael James, Wharfe Valley Community Mental Health Team Leader, and Robert F. Kehoe, Consultant Psychiatrist, Airedale General Hospital, Steeton, Keighley, West Yorkshire BD20 6TD

*Correspondence 